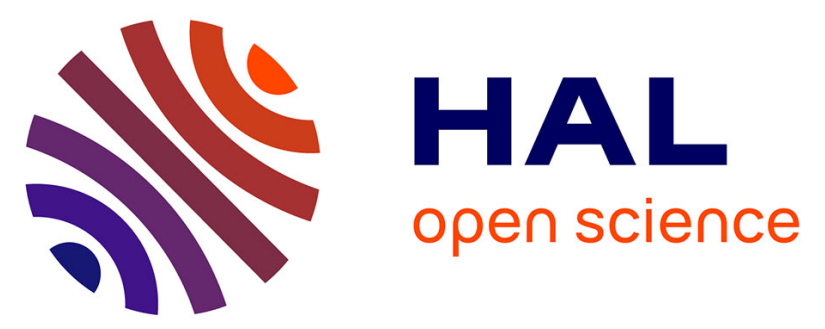

\title{
Flow Cytometric Single-Cell Analysis for Quantitative in Vivo Detection of Protein-Protein Interactions via Relative Reporter Protein Expression Measurement
}

Lina Wu, Xu Wang, Tian Luan, Jianqiang Zhang, Emmanuelle Bouveret, Xiaomei Yan

\section{To cite this version:}

Lina Wu, Xu Wang, Tian Luan, Jianqiang Zhang, Emmanuelle Bouveret, et al.. Flow Cytometric Single-Cell Analysis for Quantitative in Vivo Detection of Protein-Protein Interactions via Relative Reporter Protein Expression Measurement. Analytical Chemistry, 2017, 89 (5), pp.2782-2789. 10.1021/acs.analchem.6b03603 . hal-01788483

\section{HAL Id: hal-01788483 \\ https://hal.science/hal-01788483}

Submitted on 23 May 2018

HAL is a multi-disciplinary open access archive for the deposit and dissemination of scientific research documents, whether they are published or not. The documents may come from teaching and research institutions in France or abroad, or from public or private research centers.
L'archive ouverte pluridisciplinaire HAL, est destinée au dépôt et à la diffusion de documents scientifiques de niveau recherche, publiés ou non, émanant des établissements d'enseignement et de recherche français ou étrangers, des laboratoires publics ou privés. 
$1 \quad$ Flow Cytometric Single-Cell Analysis for Quantitative in

2 Vivo Detection of Protein-Protein Interactions via Relative Reporter Protein Expression Measurement.

$4 \quad$ Lina $\mathrm{Wu}^{\mathrm{a}, 1}, \mathrm{Xu} \mathrm{Wang}^{\mathrm{a}, 1}$, Tian Luan ${ }^{\mathrm{a}}$, Jianqiang Zhang ${ }^{\mathrm{a}}$, Emmanuelle Bouveret ${ }^{\mathrm{b}}$ and Xiaomei Yan ${ }^{\mathrm{a}, 2}$

${ }^{1}$ L. W. and X. W. contributed equally to this work.

${ }^{2}$ To whom correspondence should be addressed. E-mail: xmyan@xmu.edu.cn.

Classification:

Major: Biological Sciences 


\section{Abstract:}

Cell-based two-hybrid assays have been key players in identifying pairwise interactions, yet quantitative measurement of protein-protein interactions in vivo remains challenging. Here, we show that using relative reporter protein expression (RRPE), defined as the level of reporter expression normalized to that of the interacting protein measured in single cell, quantitative analysis of protein interactions in bacterial adenylate cyclase two-hybrid (BACTH) system can be achieved. A multicolor flow cytometer was used to measure simultanously the expression levels of one of the two putative interacting proteins and the $\beta$-galactosidase ( $\beta$-gal) reporter protein upon dual immunofluorescence staining. Single cell analysis revealed that for every bacterial culture co-transformed with the two-hybrid plasmids, there exist two cell populations with or without the expression of interacting protein and reporter protein. Using Pal and TolB protein as the model of an interacting bait and pray pair, the RRPE was found to be constant regardless of the inoculation colonies and the cultivation time. Decreased RRPE was detected for TolB mutants with two $\mathrm{N}$-terminal truncations (TolB $\Delta^{22-25}$ or TolB $\Delta^{22-33}$ ), suggesting that the RRPE was an intrinsic characteristic associated with the binding strength between the two interacting proteins. This hypothesis was verified with acid base coiled coils formed by two $\alpha$-helices of various binding affinities, for which the measured RRPE progressively decreased as the affinity decreased. Several useful applications of our RRPE-BATCH method can be expected for the quantitative detection, strength comparison, and affinity ranking of pairs of interacting proteins. 
Keywords: protein-protein interaction, yeast two-hybrid system, bacterial two-hybrid system, flow cytometry, binding affinity

\section{Significance Statement}

Assessing the intrinsic affinities for interacting proteins is of fundamental importance to explore and understand protein-protein interactions. Using a bacterial two-hybrid system (the BACTH system), we developed a quantitative method for the detection and affinity ranking of protein-protein interactions. By measuring the expression level of both the reporter protein and interacting protein at the single-cell level via immunofluorescent staining and flow cytometry, we found that the relative reporter protein expression (RRPE) is characteristic of the interacting protein pair and correlates with their binding affinity. This method can provide an efficient tool in prioritizing a large number of putative interacting proteins for following analyses. 
Protein-protein interactions are involved in virtually every cellular process, and their study is crucial in revealing protein functions, deciphering protein interaction networks, and identifying novel therapeutic targets $(1,2)$. Among numerous methodologies developed for protein interaction study, the yeast two-hybrid (Y2H) system is the most commonly used binary method for measuring direct physical interactions between two proteins, and has been estimated to account for over $50 \%$ of protein-protein interactions described in PubMed (3-5). This powerful in vivo approach interrogates two proteins, called bait and prey, one fused to a DNA-binding domain and the other fused to a transcriptional activation domain of a transcription factor and expressed in yeast. If the two proteins interact in the system, they reconstitute a functional transcription factor that induces the transcription of a reporter gene, whose output can be measured as growth of yeast colonies on selective medium or as blue coloration in a $\beta$-galactosidase ( $\beta$-gal) assay. Although the $\mathrm{Y} 2 \mathrm{H}$ system has made significant contribution to the discovery of protein-protein interactions and the interactome networks (6-8), both the false positive and false negative rates are relatively high, and all the interactions are forced to occur in the yeast nucleus and are thus not suitable for protein interaction involving membrane proteins and cytosolic proteins $(7,9)$.

To overcome the limitations of $\mathrm{Y} 2 \mathrm{H}$ system, a bacterial equivalent of the two-hybrid system was developed based on functional complementation of the catalytic domain of Bordetella pertussis adenylate cyclase (BACTH) $(10,11)$. This leads to cAMP synthesis, which in turn, triggers the expression of several resident 
genes such as the lactose or maltose operons. Particularly, this technique enabled the study of membrane proteins because cAMP is a diffusible molecule and the BACTH system does not require the hybrid proteins to be located in the nucleus as that of $\mathrm{Y} 2 \mathrm{H}$ (12). However, the BACTH system as well as the $\mathrm{Y} 2 \mathrm{H}$ system is not suitable for the quantitative measurement of pairwise protein interactions due to the lack of understanding of how the strength of the interactions correlate with the level of reconstituted reporters (13).

Flow cytometry is a well-established tool for the rapid, quantitative, and multiparameter analysis of single cells. Employing a codon-optimized yeast enhanced green fluorescent protein (yEGFP) as the reporter, Chen et al. developed a high throughput approach to study protein-protein interactions inside the cell via flow cytometric measurement (14). Through the development of a yeast surface two-hybrid (YS2H) system, Hu et al. reported quantitative flow cytometric measurement of protein-protein interactions via the secretory pathway (13). On the other hand, anchored periplasmic expression (APEx) bacterial two-hybrid system has been developed for the study of protein pairs on the basis of affinity or expression $(15,16)$. In both the YS2H and the APEx two-hybrid systems, the bait protein has to be produced at the surface of the cell, and the tag-fused prey protein has to be secreted in solution. Then, the strength of bait-pray interaction can be measured via antibody binding to the epitope tag appended to the prey protein. Compared to the surface bacterial two-hybrid system, the classic BACTH system is a well-established and much simpler approach for protein-protein interaction studies $(10,11)$. Particularly, 
the signaling cascade in the BACTH system ensures higher sensitivity for the weak and transient interactions. Therefore, a quantitative approach for the BACTH system shall greatly advance the protein-protein interaction study due to the general applicability of the BACTH system and its sensitivity for low affinity interaction.

Herein, we demonstrate that through flow cytometric detection and immunofluorescent staining, the relative reporter protein expression (RRPE), defined as the normalized report protein expression to that of the interacting protein in a single cell, can be used to quantitatively estimate the binding strength between two interacting proteins in the classical BACTH system. This feature allowed us to confirm interacting pairs of proteins, investigate determinant residues in protein-protein interaction, and compare interaction strength of different pairs. The RRPE-BACTH method described here provides a practical and powerful method for the rapid and quantitative in vivo measurement of protein-protein interactions.

\section{Results and Discussion}

Design of the BACTH System for Flow Cytometric Analysis. Scheme 1 illustrates the experimental design of the flow cytometric BACTH system. Two compatible plasmids carrying the hybrids with $\mathrm{T} 25$ and T18 domains respectively were co-transformed into the reporter strain $c^{-} a^{-}$E. coli BTH101. Between T25 or T18 domain and the hybrid proteins, His and Flag tags were inserted respectively, and were used to follow the expression of the hybrid proteins. The interaction of the hybrid proteins results in a functional complementation between T25 and T18 fragments, which reconstitutes the activity of adenylate cyclase and leads to cAMP 
synthesis. The produced cAMP interacts with the catabolite activator protein (CAP) and the cAMP/CAP complex binds to the promoter and regulates the transcription of lac $Z$ gene coding for the $\beta$-galactosidase ( $\beta$-gal) reporter expression. $\beta$-gal was specifically labeled green with rabbit-anti- $\beta$-gal antibodies and FITC-conjugated goat anti rabbit IgG. Meanwhile, anti-His/Flag mouse monoclonal antibody and DyLight 649-conjugated goat anti mouse IgG were used to label the hybrid proteins red. Upon dual immunofluorescence labeling, the bacterial sample was analyzed by flow cytometer. FITC and DyLight 649 fluorophores can be excited by the $488 \mathrm{~nm}$ and 640 $\mathrm{nm}$ lasers respectively, and the emitted green and red fluorescence signals were detected concurrently on the FL1 and FL2 fluorescence channels. Therefore, for a bacterial two hybrid sample, the expression level of both the $\beta$-gal reporter and the hybrid proteins can be detected and quantified simultaneously.
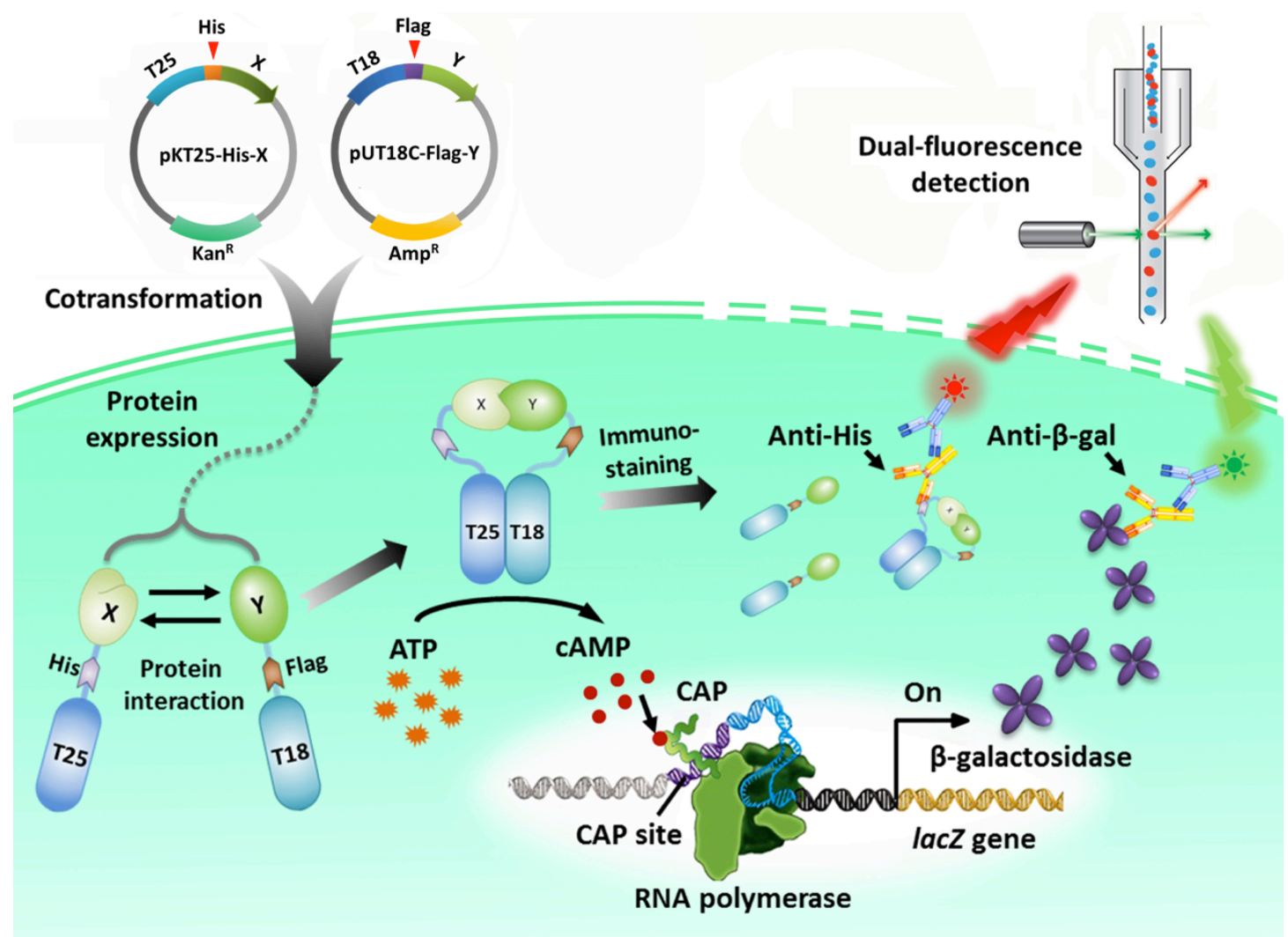
Scheme 1. Depiction of protein-protein interaction study at the single-cell level based on the BACTH system, dual immunofluorescent staining, and flow cytometric analysis.

\section{Flow Cytometric Detection of Protein-Protein Interaction by the BACTH System} via Immunofluorescent Staining of $\boldsymbol{\beta}$-gal Reporter Protein. Pal and TolB, two proteins involved in maintaining the integrity of bacterial outer membrane were chosen as the protein-protein interaction model (17). In the BACTH system, both the interacting proteins and reporter proteins are produced in the cytoplasm of bacteria, and the antibodies need to traverse the bacterial cell wall and membrane for target staining. Therefore, much efforts have been devoted to optimize the immunofluorescence labeling procedures including fixation, permeabilization, and staining (see Materials and Methods). After immunofluorescent staining of $\beta$-gal reporter protein with FITC, the samples were analyzed on the flow cytometer. Fig. 1 shows the bivariate dot-plots of side scatter intensity versus FITC fluorescence intensity obtained for E. coli BTH101 co-transformed with plasmids pUT18C-linker/pKT25-linker (negative control, no interaction), pUT18C-pal/pKT25-tolB (interacting proteins without additional tag), and pUT18C-Flag-pal/pKT25-His-tolB (tagged interacting proteins), respectively. Two distinct populations with different green fluorescence intensity were observed for bacterial samples co-transformed with plasmids containing the interacting Pal/TolB pair proteins regardless the presence of Flag/His tag or not (Fig. 1, B and C). A discriminant line between these two populations was drawn on the FL1 channel for 
157

158

159

easy discrimination, defining two regions $\mathrm{P} 1$ and $\mathrm{P} 2$. For the negative control sample, approximately $94.8 \%$ of the cells fall in the P1 region (Fig. 1A), whereas for cells co-transformed with plasmids containing the interacting proteins with or without tags, about $34.8 \%$ and $42.4 \%$ of the cells fall in the $\mathrm{P} 2$ region. The fluorescence distribution histograms of these three samples are plotted in Fig. 1D. Comparable median fluorescence intensities (MFI) for cells falling in the P1 region were observed for all three samples, suggesting that for bacterial culture co-transformed with two plasmids carrying Pal and TolB genes, there exists a large fraction of cells (about 40\%) in which the $\beta$-gal reporter protein cannot be detected. Meanwhile, events residing in the $\mathrm{P} 2$ region can be ascribed to cells that co-express $\mathrm{Pal}$ and TolB interacting proteins inside a single cell which leads to the expression of $\beta$-gal reporter. Because the interaction between Pal and TolB is robust and well characterized, the fraction of the cells that are similar to the negative control may be ascribed to the lack of expression of either one or both of the interacting proteins. As plasmid loss is excluded by the use of antibiotics in the culture media, this all-or-nothing phenomenon can only be explained by the bistability in the lactose utilization network of E. coli. In the BACTH system, the reporter gene and hybrid plasmids are both regulated by the wild type Plac promoter (18). For cells falling in the $\mathrm{P} 2$ region, the MFI are 3135 and 2539 for bacterial cultures transformed with interacting protein genes without and with Flag/His tag, respectively, suggesting that tag insertion to the C-terminal of the two proteins did not prevent the interaction. The observation of two populations with completely different behaviors regarding reporter $\beta$-gal expression 
highlights the importance and need of single-cell analysis for the BACTH system. In contrast to the ensemble-averaged measurement by spectrophotometers, flow cytometric analysis can reveal the inherent heterogeneity of bacterial populations in $\beta$-gal expression that will provide more insights into the BACTH system.

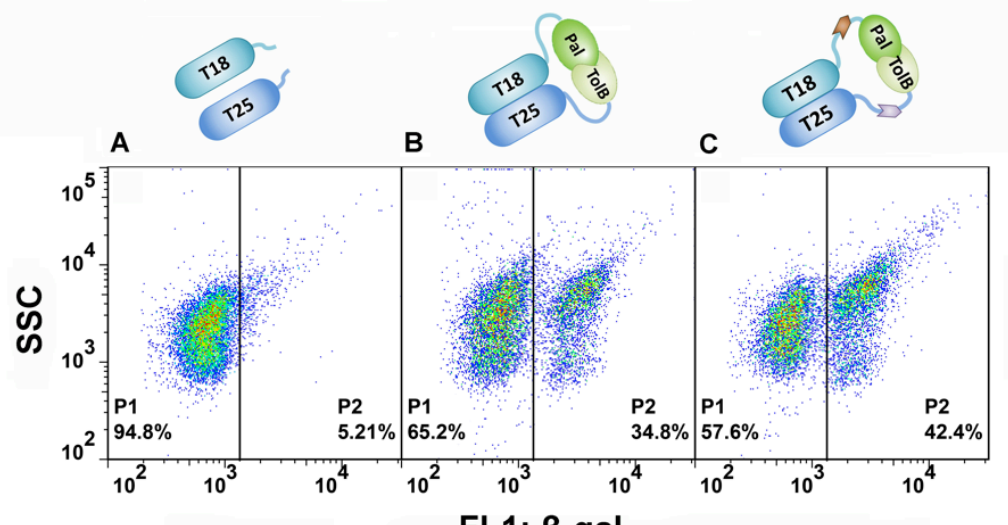

FL1: $\beta$-gal

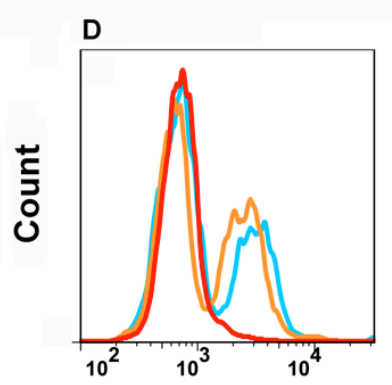

FL1: $\beta$-gal

Fig. 1. Flow cytometric analysis of protein-protein interaction by the BACTH system upon $\beta$-gal immunofluorescent staining. Bivariate dot-plots of side scatter intensity (SSC) versus FITC fluorescence intensity (FL) for E. coli BTH101 co-transformed with plasmids of A) pUT18C-linker/pKT25-linker, B) pUT18C-pal/pKT25-tolB, and C) pUT18C-Flag-pall pKT25-His-tolB, respectively. D) FITC green fluorescence distribution histograms of $\beta$-gal for E. coli BTH101 with no interaction (red, case A), with expression of interacting proteins (blue, case B), and with expression of tagged interacting (orange, case $\mathrm{C}$ ).

\section{Simultaneous Measurement of the Expression of Interacting Protein and}

Reporter Protein. In order to study the relationship between the expression of $\beta$-gal reporter protein and the expression of hybrid proteins, the Flag or His tag of one interacting protein and the $\beta$-gal reporter protein were immunofluorescently labeled with Dylight-649 and FITC, respectively. DyLight-649 was chosen to label the tag fragment because it can be efficiently excited by the $640 \mathrm{~nm}$ laser, which avoids 
spectral cross-talk with FITC. The green and red fluorescence signals were detected on the FL1 and FL2 channel, respectively. Figs. 2A and 2B show the bivariate dot-plots of $\beta$-gal reporter protein versus TolB expression (via His tag labeling) or Pal expression (via Flag tag labeling), respectively. Quadrant gates were created for all the samples. For the negative control with E. coli BTH101 co-transformed with pUT18C-linker/pKT25-linker (no interacting protein expression), most cells $(94.0 \%$ and 93.9\%) fall into the Q4 region (Figs. 2A1 and 2B1) with negligible fluorescence on both the green and red fluorescence channels. For E. coli BTH101 co-transformed with plasmids encoding interacting proteins but without tags (pUT18C-pal/pKT25-tolB), 32.6\% and 32.0\% of the cells fall into the Q1 region due to the expression of $\beta$-gal reporter protein. The two population phenomenon is similar to the one observed in Fig. 1. For E. coli BTH101 co-transformed with plasmids encoding interacting proteins with tags (pUT18C-Flag-pal/pKT25-His-tolB), 53.6\% and $54.4 \%$ of the cell population resides in the Q4 region (neither the expression of $\beta$-gal nor the expression of interacting protein), while $42.5 \%$ and $41.2 \%$ of the cells fall in the Q2 region indicating concurrent expression of the interacting proteins and the $\beta$-gal reporter (Figs. 2A3 and 2B3). The similar ratios of Q2 reveal that only the co-expression of the interacting proteins leads to the expression of the reporter gene. Because expression of all of them are driven by cAMP in a positive feedback loop, this establishes bistability. Clearly, using dual immunofluorescence staining, simultaneous measurement of interacting protein expression and protein-protein interaction (via reporter protein expression) can be successfully achieved at the 
single-cell level using flow cytometry. It is worthy to note that although

pUT18C-Flag-pal and pKT25-His-tolB are two compatible plasmids, they have distinct replication origins and different copy numbers (11). The plasmid of lower copy number (pKT25-His-tolB) normally results in a lower level of protein expression, which determines the rate of complex formation with its interacting protein partner.

Note that the same PMT voltage was used for the detection of His-tag and Flag-tag signals, and the relatively higher signal for His tag is due to the higher affinity of anti-his antibody.
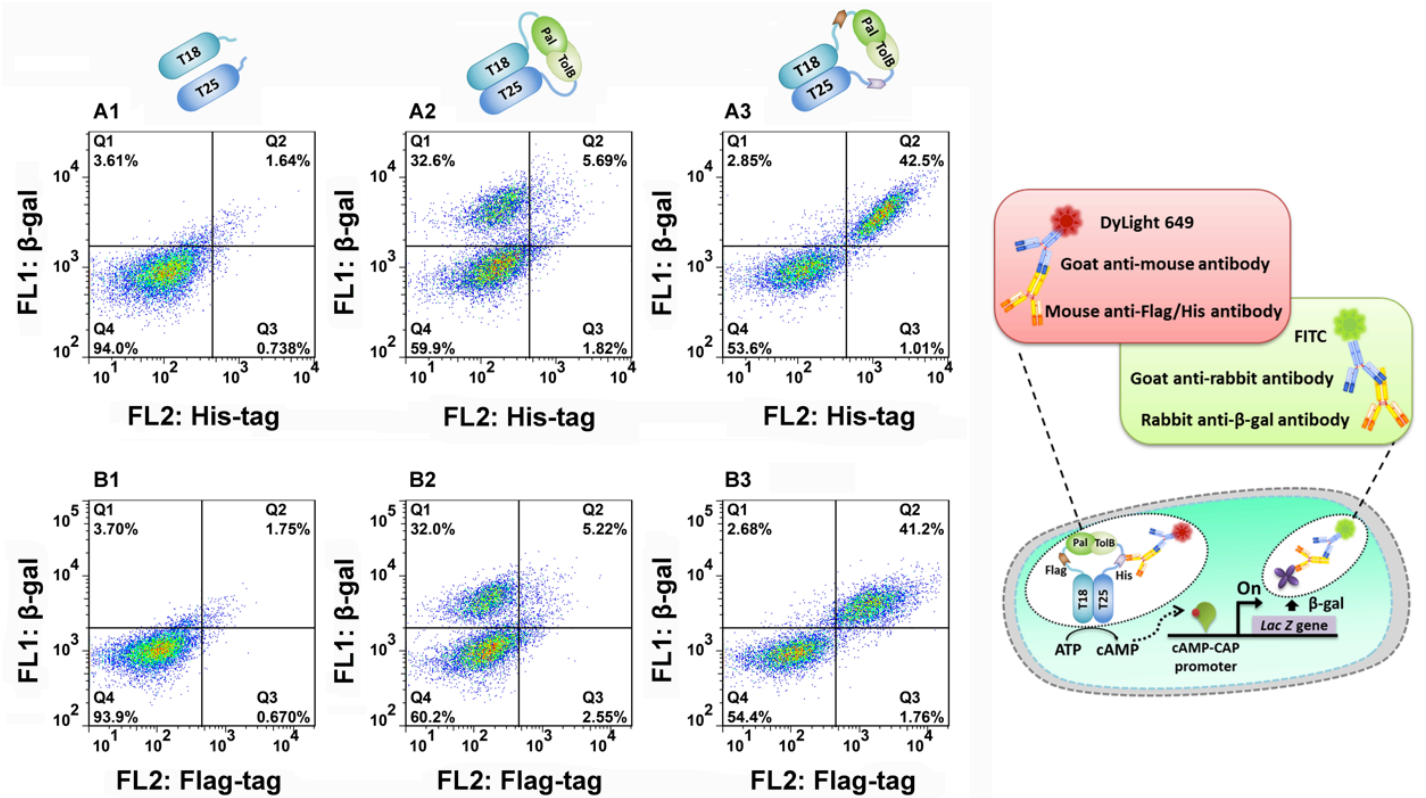

Fig. 2. Simultaneous measurement of the expression of interacting proteins and reporter protein by flow cytometry. (A) Bivariate dot-plot of green fluorescence intensity ( $\beta$-gal) versus red fluorescence intensity (His-tag) for E. coli BTH101 co-transformed with plasmids of A1) pUT18C/pKT25, A2) pUT18C-pal/pKT25-tolB, and A3) pUT18C-Flag-pal/pKT25-His-tolB, respectively. (B) Bivariate dot-plot of green fluorescence intensity ( $\beta$-gal) versus red fluorescence intensity (Flag-tag) for $E$. coli BTH101 co-transformed with plasmids of B1) pUT18C/pKT25, B2) pUT18C-pal/pKT25-tolB, and B3) pUT18C-Flag-pal/pKT25-His-tolB, respectively. 
Correlation between the Protein Interaction Strength and the Expression of Interacting Proteins in a Single Cell. As illustrated in Figure 1, in the BACTH system, expression of the $\beta$-gal reporter protein is regulated by the production of cAMP and thus by the interaction of two hybrid proteins (10). We examined the relationship between the $\beta$-gal reporter protein expression and His-TolB (the plasmid with lower copy number in the cell) expression by flow cytometry. When the cultures of $E$. coli $\mathrm{BTH} 101$ reached a sufficient cell density (OD $\sim 1.5)$ after 12 h cultivation, these cultures were co-transformed with plasmids of pUT18C-Flag-pal/pKT25-His-tolB every two hours. Before immunofluorescent staining, $\beta$-gal activity of each sample was assayed by a classical Miller's protocol on a spectrophotometer. Fig. 3A shows that $\beta$-gal activity increased from 375 at $12 \mathrm{~h}$ to 702 at $16 \mathrm{~h}$ and started to decrease slowly after then. Meanwhile, single cell measurements by flow cytometry indicate that with the increase of cultivation time from $12 \mathrm{~h}$ to $20 \mathrm{~h}$, the fraction of cells expressing $\beta$-gal and His-TolB (Fig. 3B) kept increasing from $7.5 \%$ to $75.5 \%$. In contrast, the MFIs of positive cells decreased from 14500 to 2508 for $\beta$-gal signal (Fig. 3B1) and from 4155 to 495 for His-TolB signal (Fig. 3B2), respectively. This phenomenon could be explained by the dilution of the proteins inside a single cell upon cell division (19). When we plotted the MFI of $\beta$-gal versus that of His-TolB after background signal subtraction for each protein, i.e. $\left(\mathrm{MFI}_{\beta-\mathrm{gal}}, \mathrm{P}_{2}-\mathrm{MFI}_{\beta-\mathrm{gal}}, \mathrm{P} 1\right)$ versus $\left(\mathrm{MFI}_{\mathrm{His}-\mathrm{TolB}, \mathrm{P} 2}-\mathrm{MFI}_{\mathrm{His}-\mathrm{TolB}}, \mathrm{P} 1\right)$, a linear correlation with $\mathrm{R}^{2}$ of 0.9995 was obtained (Fig. 3C), which suggests that in the BACTH system, expression of the $\beta$-gal reporter protein is linearly proportional to the expression of 
260
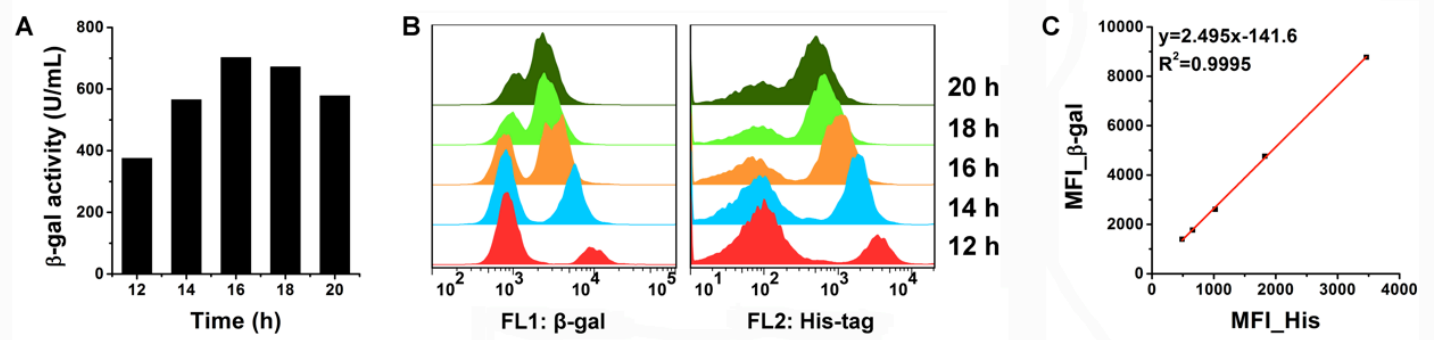

Fig. 3. Enzymatic and flow cytometric analysis of the reporter protein $\beta$-gal for $E$. coli BTH101 co-transformed with plasmids pUT18C-Flag-pal/pKT25-His-tolB at different cultivation time. A) Column chart of $\beta$-gal activity measured with a classical Miller's assay. B) Histograms of the fluorescence intensity distribution for $\beta$-gal and His-TolB measured by flow cytometry. C) The correlation curve between the median fluorescence intensities of $\beta$-gal and His-TolB after background subtraction.

The heterogeneity in the BACTH system has been well recognized because there exist a big difference in plasmid copy number in different bacterial cells (11) and stochasticity inherent in the biochemical process of gene expression (20). In order to validate the generality of this observation, we first examined the correlation between the expression of $\beta$-gal reporter protein and interacting protein for different colonies at the same cultivation time. Fifteen different colonies were randomly picked from the culture plate of E. coli BTH101 co-transformed with plasmids pUT18C-Flag-pal/pKT25-His-tolB and inoculated in separate LB broth and cultivated for $16 \mathrm{~h}$. Fig. 4A shows the plot of $\beta$-gal expression versus that of interacting protein His-TolB for different colonies, and a linear correlation was observed with $\mathrm{R}^{2}$ of 0.9789. These data along with those reported in Fig. 3 suggest that for an interacting protein pair, there is an important heterogeneity in the expression of both the hybrid 
and reporter proteins, but that the expression of $\beta$-gal reporter protein exhibits a linear proportion to that of the hybrid proteins regardless of different cultivation time for a single colony or different colonies at the same cultivation time. Clearly, these results demonstrate that the expression of the $\beta$-gal reporter protein is not only affected by the affinity of the interacting protein pair but also by the expression level of the hybrid proteins. Therefore, we propose to use relative reporter protein expression (RRPE), defined as the normalized $\beta$-gal expression to that of the interacting protein, to estimate the interaction strength of protein pairs. As shown in Fig. 4B, the measured RRPE (blue dots) for the Pal-TolB interaction pair exhibits a constant value, whereas the $\beta$-gal activity (red dots) is much more diverging among different colonies.

A

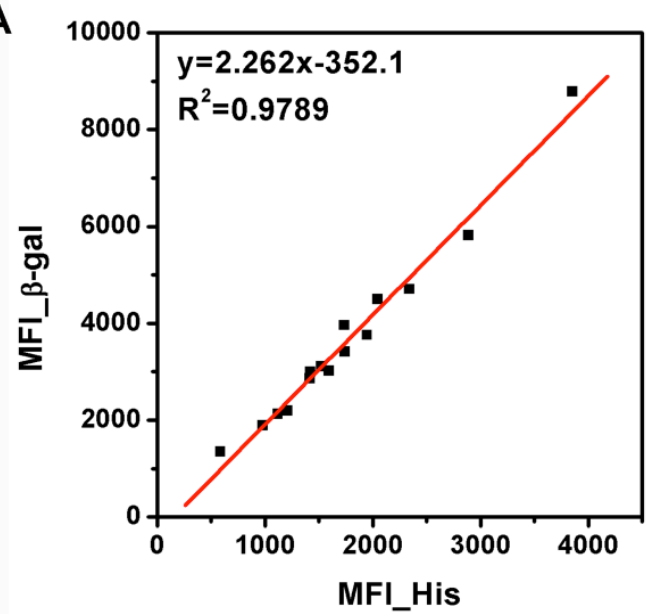

B

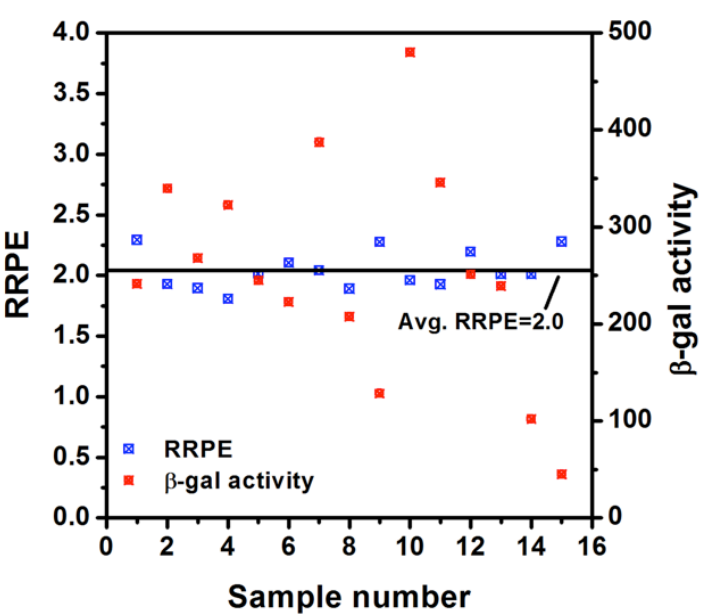

Fig. 4. Flow cytometric analysis of the expression of $\beta$-gal reporter protein and interacting protein His-TolB for bacterial samples inoculated with different single colonies. A) The correlation curve between the median value of $\beta$-gal fluorescence intensity and that of His-TolB. B) Plot of the relative reporter protein expression (RRPE) versus $\beta$-gal activity for 15 bacterial cultures inoculated with different single colonies randomly picked from the plate co-transformed with plasmids pUT18C-Flag-pal/pKT25-His-tolB. 


\section{Validation of the RRPE-BACTH Method for the Measurement of Protein}

Interaction Strength. To investigate the potential of using RRPE-BACTH method for evaluating the strength of protein-protein interactions, we compared the Pal-TolB interaction by using two mutated forms of TolB along with the wild type TolB. These two mutated TolB proteins bear truncations, one with four residues deleted from the $\mathrm{N}$-terminus (TolB $\Delta^{22-25}$ ) and the other with the entire N-terminal sequence deleted (TolB $\Delta^{22-33}$ ), which lower the binding affinity to Pal (21). The dissociation constants $\mathrm{K}_{\mathrm{D}}$ of these two truncated TolB proteins with Pal were reported to be $313 \pm 15 \mathrm{nM}$ and $337 \pm 18 \mathrm{nM}$, respectively, via ITC measurement at $30^{\circ} \mathrm{C}$, which are about tenfold higher than that of the wild type TolB $(38 \pm 3 \mathrm{nM})$. E. coli $\mathrm{BTH} 101$ cells were co-transformed with pUT18C-Flag-pal/pKT25-His-tolB, pUT18C-Flag-pal/pKT25-His-tolB $\Delta^{22-25}$, or pUT18C-Flag-pal/pKT25-His-tolB $\Delta^{22-33}$ plasmids and plated. Three individual colonies were picked and inoculated into LB broth for each protein pair. These samples were immunofluorescence stained and analyzed on the flow cytometer. Fig. 5A shows the representative bivariate dot-plots of $\beta$-gal green fluorescence versus His-TolB red fluorescence and their fluorescence distribution histograms for these three pairs. Fig. 5B indicates that the wild type TolB exhibits the highest RRPE of $2.04 \pm 0.21$, and the mutated TolB $\Delta^{22-25}$ and TolB $\Delta^{22-33}$ shared comparable RRPE values of $0.88 \pm 0.07$ and $0.91 \pm 0.06$, respectively. Hence, the RRPE value follows the change in binding affinity of the TolB/Pal interaction, with larger RRPE corresponds to higher binding affinity in the BACTH system. Therefore, RRPE may be used to assess the binding affinity of protein-protein 
interaction in the BACTH system.
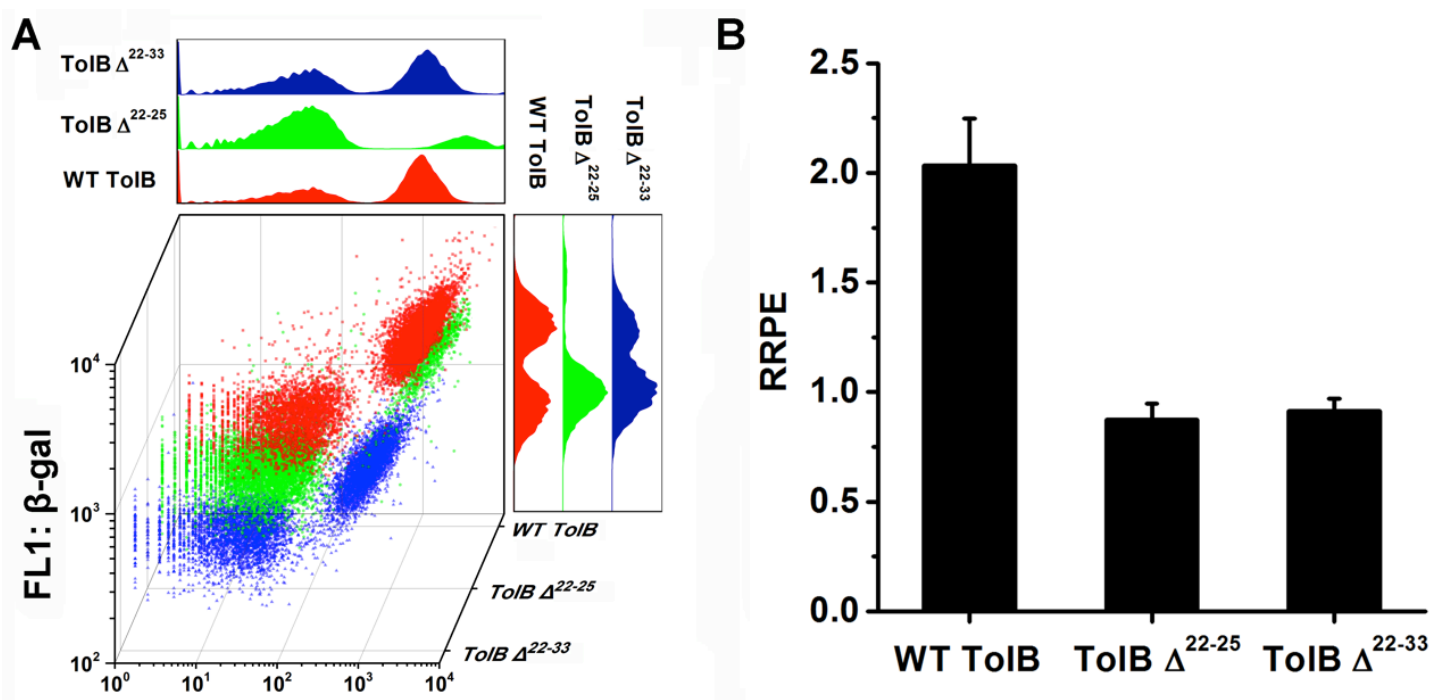

FL2: His-tag

Fig. 5. RRPE measurement for Pal interacting with TolB and the two TolB mutants by the BACTH system and flow cytometry. (A) The bivariate dot-plots of $\beta$-gal green fluorescence versus His-TolB red fluorescence and the fluorescence distribution histograms for wild type TolB and its two truncated forms: TolB $\Delta^{22-25}$ and TolB $\Delta^{22-33}$. (B) Column chart of the RRPE for Pal interaction with TolB and the two truncated forms. The error bar represents the standard deviation of three replicates.

To further validate the applicability of the RRPE-BACTH method in affinity assessment, five pairs of acid (En) and base (Kn) $\alpha$-helices with various heptad repeats (n) that associate into coiled coils were constructed into the BACTH system (Fig. 6A). E coil and $\mathrm{K}$ coil interacts through hydrophobic interaction at the interface and electrostatic attraction between the oppositely charged residues from the helix, and higher affinity is associated with longer helix (22). Among the five coiled-coils chosen in the present study, the dissociation constants $\left(K_{D}\right)$ measured by surface plasmon resonance $(\mathrm{SPR})$ using a BIAcore were $30000 \pm 3000,7000 \pm 800,116 \pm 8$, $14 \pm 1$, and $0.063 \pm 0.005 \mathrm{nM}$ for the interactions of E3-K3, E5-K3, E4-K4, E5-K4, 
and E5-K5, respectively (22). E. coli BTH101 cells were co-transformed with pUT18C-Flag-En/pKT25-His-Kn plasmids and plated. For each protein pair, three individual colonies were picked and inoculated into LB broth separately. These samples were immunofluorescence stained and analyzed on the flow cytometer. Fig. 6B shows the representative bivariate dot-plots of $\beta$-gal green fluorescence versus His-Kn red fluorescence along with the fluorescence distribution histograms for these five interaction pairs. The measured RRPE values were $3.8 \pm 0.1,6.4 \pm 0.5,9.9 \pm 0.1$, $11.8 \pm 0.3$, and $5.9 \pm 0.6$ for E3-K3, E5-K3, E4-K4, E5-K4, and E5-K5, respectively. It should be noted that taking advantage of high sensitivity of the RRPE-BATCH method, we can discriminate interactions with $\mathrm{K}_{\mathrm{D}}$ lower than $10^{4} \mathrm{nM}$. Fig. $6 \mathrm{C}$ shows that the measured RRPE exhibited a strong correlation with the interaction affinity from E3-K3 to E5-K4 except for E5-K5. This could be explained by the fact that E5-K5 fits an interacting model with a relatively fast association and a very slow dissociation, which is different from the other pairs (22). In contrast, in the BACTH system, the synthesis of cAMP is irreversible and depends mainly on the rate at which two coils associate (on-rate) to initiate complementation of T25 and T18. Because the E5 coil was fused to T18 or T25 domain in the BATCH system, the on-rate of binding with $\mathrm{K} 5$ could be reduced which leads to a lower binding affinity as demonstrated with a decreased RRPE value (22). However, it needs to be pointed out that the proposed RRPE method does not allow for the absolute quantification of protein interaction affinity, because the BACTH system itself is an indirect method to detect protein-protein interaction. Nonetheless, the good correlation between the measured 
361 protein variants in a given interacting pair of proteins.

\section{A}

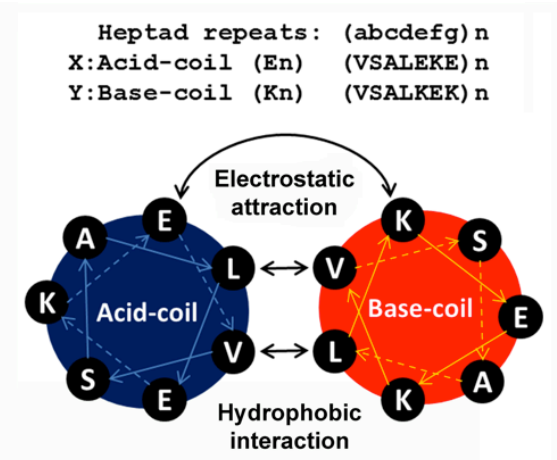

C

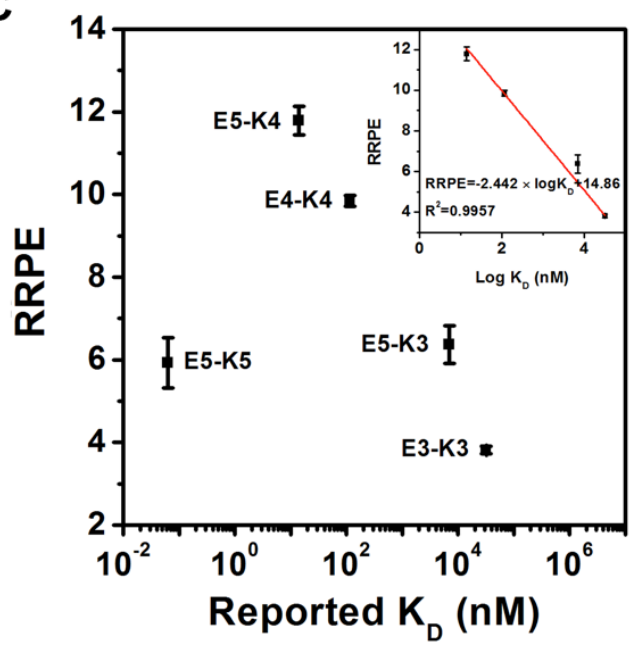

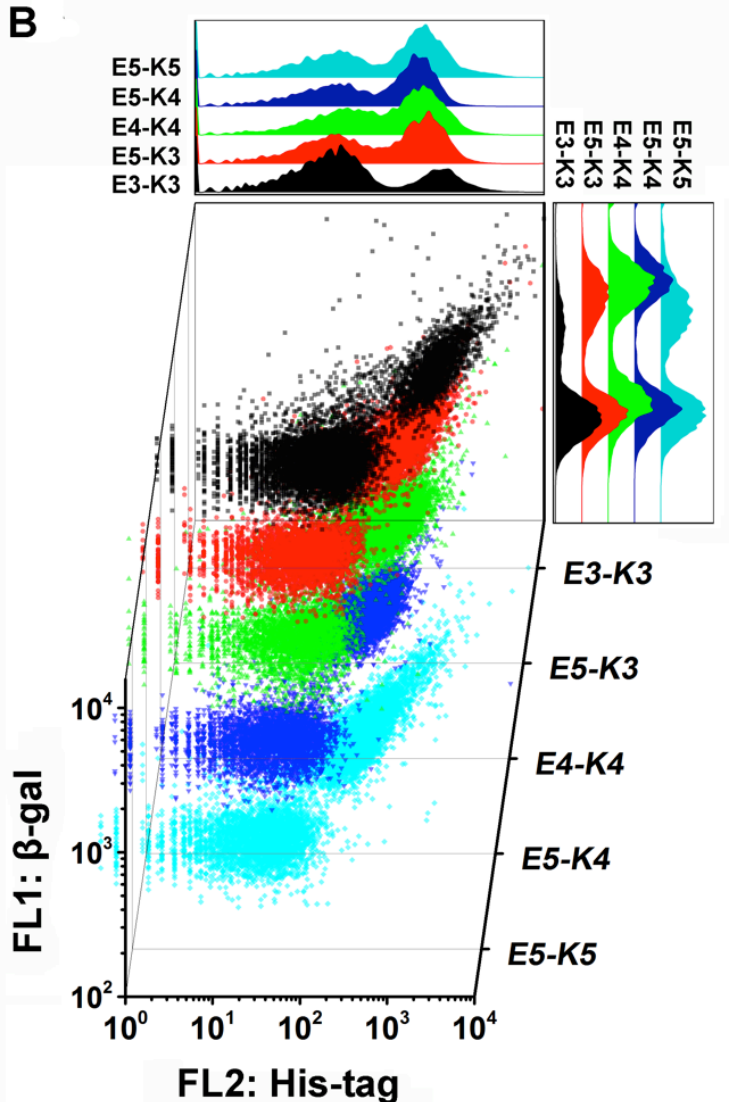

362

Fig. 6. RRPE measurement for five pairs of coiled coil interactions using the BACTH system and flow cytometry. (A) A schematic (adapted from the Fig. 1 by De Crescenzo et al. (22)) of the acid (En)-base (Kn) coiled coils interaction with $n$ indicating the number of heptad repeats. (B) The bivariate dot-plots of $\beta$-gal green fluorescence versus His tag red fluorescence intensity for E. coli BTH101 cells co-transformed with plasmids of pUT18C-Flag-En/pKT25-His-Kn. (C) The correlation of the RRPE values measured by flow cytometry for coiled coil interactions occurring in the BACTH system with the affinity measured by SPR (22). The error bar represents the standard deviation of three replicates.

\section{Conclusion}


We have developed a sensitive in vivo method for the quantitative measurement of protein-protein interaction via the BACTH system and flow cytometry. Taking advantage of the high-throughput and multiparameter measurement of single cell by flow cytometry, the expression of reporter protein and interacting proteins can be simultaneously measured and correlated at the single-cell level. It was found out that for the BATCH system, for a bacterial culture inoculated even with a single colony co-transformed with two plasmids encoding each of the two interacting proteins, there exist two populations and a large heterogeneity for each population in protein expression and reporter protein production, which would otherwise be masked by the ensemble-averaged measurements. By measuring the expression level of interacting protein and reporter protein for the population expressing $\beta$-gal, it was identified that for an interacting protein pair, the value of RRPE is constant and is an intrinsic feature. Moreover, a good correlation of the RRPE with the binding affinity of protein pair was observed for Pal-TolB interaction with WT and mutant TolB proteins and for several coiled-coil interactions. The RRPE method proposed here can not only be used to validate existing protein interaction and finding new ones, but also to rank the strength of interaction. It may further be used for highthroughput study of the binding site of protein-protein complexes, selection of high-affinity antibodies, and screening of peptide inhibitor libraries.

\section{Materials and Methods}

Reagents and Chemicals. Rabbit anti- $\beta$-galactosidase $\operatorname{IgG}$ was purchased from 
395

396

397

398

399

400

401

402

403

404

405

406

407

408

409

410

411

412

413

414

415

416

Molecular Probes (Eugene, OR, USA). FITC-conjugated anti-His mouse monoclonal antibody and goat anti-rabbit $(\mathrm{GAR}) \operatorname{IgG}(\mathrm{H}+\mathrm{L})$ were obtained from TransGen Biotech (Beijing, China). DyLight-649-conjugated goat anti-mouse (GAM) IgG $(\mathrm{H}+\mathrm{L})$ was purchased from EarthOx (San Francisco, CA, USA). Antibodies were diluted in 1\% fetal bovine serum (FBS) (obtained from Hyclone, Logan, Utah, USA) freshly prepared in PBS before use. Enzymes used for molecular cloning were obtained from TaKaRa Biotech (Dalian, China). Ortho-nitrophenyl- $\beta$-galactoside (ONPG), lysozyme, GTE (50 mM Glucose, 25 mM Tris, 10 mM EDTA, pH 8.0), and X-gal were purchased from Sangon Biotech (Shanghai, China). Paraformaldehyde (PFA) stock solution (16\%) was obtained from Alfa Aesar (Ward Hill, MA, USA). Other reagents were purchased from Sinopharm Chemical Reagent (Shanghai, China). All the buffers were filtered through a $0.22 \mu \mathrm{m}$ filter and used within three weeks.

Bacterial Strains and Plasmids. E. coli ER2738 was used for the cloning experiments. The recombinant plasmids used in the present study are summarized in Table S1 and were verified by sequencing. Oligonucleotides were synthesized by Sangon Biotech and are listed in Table S2. Plasmid pKT25-His-tolB was constructed by inserting the histidine tag (His-tag) into pEB362 at the PstI/EcoRI sites. The pal gene was digested with EcoRI and XhoI from pEB356 and inserted into the EcoRI/XhoI sites of pEB1030 to produce pUT18C-Flag-pal. Genes of tolB $\Delta^{22-25}$ and tolB $\Delta^{22-33}$ were amplified from plasmid pEB362 using suitable primers listed in Table S2. The PCR products were cleaved by EcoRI and XhoI and cloned into the EcoRI/XhoI sites of pKT25-His to yield plasmids pKT25-His-tolB $\Delta^{22-25}$ and 
pKT25-His-tolB $\Delta^{22-33}$. To introduce $\mathrm{Kn}$ and En sequences into each BACTH vectors, oligonucleotide primers of $\mathrm{Kn}$ and En that are complementary to each other were synthesized as listed in Table S2 and annealed by heating at $95{ }^{\circ} \mathrm{C}$ for $5 \mathrm{~min}$. Followed by cooling to room temperature, the products with cohesive ends were inserted into plasmids pKT25-His and pUT18C-Flag at the EcoRI/XhoI sites to obtain plasmids pKT25-His-Kn and pUT18C-Flag-En, respectively.

E. coli BTH101 (F-, cya-99, araD139, galE15, galK16, rpsL1, hsdR2, mcrA1, $m c r B 1)$ was used as the reporter strain of the BACTH system. Competent E. coli BTH101 strains co-transformed with two-hybrid plasmids bearing two different antibiotic resistances were spread on Luria-Bertani (LB) plates containing $100 \mu \mathrm{g} / \mathrm{mL}$ ampicillin, $50 \mu \mathrm{g} / \mathrm{mL}$ kanamycin, and $40 \mu \mathrm{g} / \mathrm{mL} \mathrm{X-gal} \mathrm{at} 30^{\circ} \mathrm{C}$. After incubating the plates for 2 days, single colonies with successful co-transformation of two hybrid plasmids were picked and inoculated in $2 \mathrm{~mL}$ of LB containing $100 \mu \mathrm{g} / \mathrm{mL}$ ampicillin and $50 \mu \mathrm{g} / \mathrm{mL}$ kanamycin. Cultures were grown overnight with shaking (250 rpm) at $30{ }^{\circ} \mathrm{C}$, unless specified otherwise. The harvested bacterial sample was adjusted to $\mathrm{OD}_{600} \sim 1.0$, immunofluorescently stained and analyzed on the flow cytometer.

Immunofluorescent Staining. To a $200 \mu \mathrm{L}$ of the harvested bacterial cells, $8 \mu \mathrm{L}$ of 1 $\mathrm{M} \mathrm{NaPO}_{4}(\mathrm{pH}$ 7.4) and $40 \mu \mathrm{L}$ of the primary fixative buffer (3 $\mu \mathrm{L}$ of $25 \%$ gluteraldehyde per $\mathrm{mL}$ of $16 \%$ paraformaldehyde) were added and incubated at room temperature for $15 \mathrm{~min}$ followed by $30 \mathrm{~min}$ on ice. The sample was washed twice with $200 \mu \mathrm{L}$ PBS and resuspended in $50 \mu \mathrm{L}$ PBS. Then, $500 \mu \mathrm{L}$ of ice cold $80 \%$ methanol was added and the sample was treated for $1 \mathrm{~h}$ at room temperature. The 
sample was washed twice with GTE buffer. The cells were permeabilized by resuspending in $100 \mu \mathrm{L}$ of $2 \mathrm{mg} / \mathrm{mL}$ lysozyme in GTE and incubated for $10 \mathrm{~min}$ at room temperature. After washing twice with PBS, the cells were blocked in $100 \mu \mathrm{L} 1 \%$ FBS for $10 \mathrm{~min}$. Then $20 \mu \mathrm{L}$ of the suspension was centrifuged and resuspended in 40 $\mu \mathrm{L}$ of $5 \mu \mathrm{g} / \mathrm{mL}$ rabbit anti- $\beta$-gal antibody with/without $5 \mu \mathrm{g} / \mathrm{mL}$ mouse anti-His/Flag antibody depending on the experimental requirement. After $1 \mathrm{~h}$ incubation at room temperature, the sample was centrifuged and washed with PBS, then resuspended in $40 \mu \mathrm{L}$ of $10 \mu \mathrm{g} / \mathrm{mL}$ FITC-conjugated GAR antibody with/without DyLight-649-conjugated GAM antibody. The suspension was incubated for $30 \mathrm{~min}$ at room temperature, centrifuged, and resuspended in $50 \mu \mathrm{L}$ PBS. For flow cytometry analysis, the sample should be diluted 500-fold with PBS before loading.

Flow Cytometric Measurement. A Becton Dickinson FACSVerse flow cytometer equipped with $488 \mathrm{~nm}$ and $640 \mathrm{~nm}$ excitation lasers was used in this study. FL1 (527/32 nm band-pass filter) channel and FL2 (660/10 nm band-pass filter) channel were used to detect the fluorescence of FITC and DyLight 649, respectively for the immunofluorescently stained bacteria. A threshold value of 200 was set on FL1 to eliminate non-bacterial particles. A total of 10000 events falling in the gated region were collected for each sample. Data acquisition and analysis were carried out by using BD FACSuite software. The data were analyzed by Flowjo 7.6.1 software (Tree Star, Inc., Ashland, OR).

Measurement of $\beta$-Gal Enzyme Activity by ONPG Colorimetric Assay. A protocol described in the literature was followed (12). Briefly, $200 \mu \mathrm{L}$ of the 
461

462

463

464

465

466

467

468

469

470

471

472

473

474

475

476

477

478

479

480

481

482

harvested bacterial cells $\left(\mathrm{OD}_{600} \sim 1.0\right)$ were treated by $3 \mu \mathrm{L}$ toluene and $3 \mu \mathrm{L} 0.01 \%$ SDS (shaking at $37^{\circ} \mathrm{C}$ for $\left.30 \mathrm{~min}\right)$. Then $1.8 \mathrm{~mL}$ PM2 buffer $\left(70 \mathrm{mM} \mathrm{Na} 2 \mathrm{HPO}_{4} \cdot 12\right.$

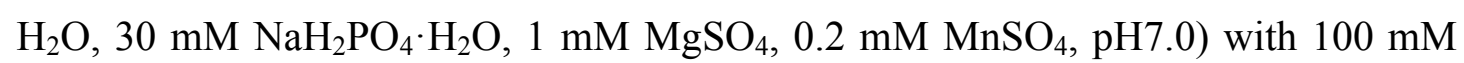
$\beta$-mercaptoethanol was added and mixed thoroughly. After that, $250 \mu \mathrm{L}$ of the ONPG substrate solution ( $4 \mathrm{mg} / \mathrm{mL}$ ONPG in PM2 buffer without $\beta$-mercaptoethanol) was added to $1 \mathrm{~mL}$ of the mixture. The enzymatic reaction was carried out immediately on a DU-800 spectrophotometer (Beckman Coulter) with measurement of $\mathrm{OD}_{420 \mathrm{~nm}}$. The $\beta$-galactosidase activity corresponds to $200 \times\left(\mathrm{OD}_{420} \mathrm{~nm}, \mathrm{t2}-\mathrm{OD}_{420 \mathrm{~nm}, \mathrm{t1}}\right) /(\mathrm{t} 2-\mathrm{t} 1)(\mathrm{min})$ $\times 10$. The factor 200 is the inverse of the absorption coefficient of o-nitrophenol, while the factor 10 is the dilution factor.

\section{ACKNOWLEDGEMENTS}

We acknowledge support from the National Natural Science Foundation of China (21105082, 21225523, 91313302, 21027010, 21475112, 21472158, and 21521004), and the Program for Changjiang Scholars and Innovative Research Team in University (IRT13036), for which we are most grateful.

\section{Author contributions}

L.W., X.W., and X.Y. conceived and designed the research; L.W., X.W., T.L., and J.Z. performed research; L.W., X.W., and X.Y. analyzed data and wrote the paper.

\section{Conflict of interest statement}

The authors declare no conflict of interest. 


\section{References:}

484 1. Bonetta L (2010) Protein-protein interactions: Interactome under construction. Nature

8. Vidal M \& Fields S (2014) The yeast two-hybrid assay: still finding connections after 25 years. Nature Methods 11(12):1203-1206.

9. Stellberger T, et al. (2010) Improving the yeast two-hybrid system with permutated fusions proteins: the Varicella Zoster Virus interactome. Proteome Sci 8:8.

10. Karimova G, Pidoux J, Ullmann A, \& Ladant D (1998) A bacterial two-hybrid system based on

11. Battesti A \& Bouveret E (2012) The bacterial two-hybrid system based on adenylate cyclase 12. Karimova G, Dautin N, \& Ladant D (2005) Interaction network among Escherichia coli membrane proteins involved in cell division as revealed by bacterial two-hybrid analysis. Journal of Bacteriology 187(7):2233-2243.

13. Hu X, Kang S, Chen X, Shoemaker CB, \& Jin MM (2009) Yeast surface two-hybrid for quantitative in vivo detection of protein-protein interactions via the secretory pathway. $J$ Biol Chem 284(24):16369-16376.

14. Chen J, et al. (2008) A yEGFP-based reporter system for high-throughput yeast two-hybrid assay by flow cytometry. Cytometry $A$ 73(4):312-320.

15. Harvey BR, et al. (2004) Anchored periplasmic expression, a versatile technology for the isolation of high-affinity antibodies from Escherichia coli-expressed libraries. Proc Natl Acad Sci U S A 101(25):9193-9198.

16. Jeong KJ, Seo MJ, Iverson BL, \& Georgiou G (2007) APEx 2-hybrid, a quantitative protein-protein interaction assay for antibody discovery and engineering. Proc Natl Acad Sci U S A 104(20):8247-8252.

17. Bonsor DA, Grishkovskaya I, Dodson EJ, \& Kleanthous C (2007) Molecular mimicry enables competitive recruitment by a natively disordered protein. Journal of the American Chemical Society 129(15):4800-4807.

18. Ozbudak EM, Thattai M, Lim HN, Shraiman BI, \& van Oudenaarden A (2004) Multistability in 
the lactose utilization network of Escherichia coli. Nature 427(6976):737-740.

19. Roostalu J, Joers A, Luidalepp H, Kaldalu N, \& Tenson T (2008) Cell division in Escherichia coli cultures monitored at single cell resolution. BMC Microbiol 8:68.

20. Elowitz MB, Levine AJ, Siggia ED, \& Swain PS (2002) Stochastic gene expression in a single cell. Science 297(5584):1183-1186.

21. Bonsor DA, et al. (2009) Allosteric beta-propeller signalling in TolB and its manipulation by translocating colicins. Embo Journal 28(18):2846-2857.

22. De Crescenzo G, Litowski JR, Hodges RS, \& O'Connor-McCourt MD (2003) Real-time monitoring of the interactions of two-stranded de novo designed coiled-coils: effect of chain length on the kinetic and thermodynamic constants of binding. Biochemistry 42(6):1754-1763.

537 